\title{
Correction to: Interdependence of laforin and malin proteins for their stability and functions could underlie the molecular basis of locus heterogeneity in Lafora disease
}

\author{
Shuchi Mittal ${ }^{1,2}$, Mamta Upadhyay ${ }^{1}$, Pankaj Kumar Singh ${ }^{1,3}$, Rashmi Parihar ${ }^{l}$ \\ and Subramaniam Ganesh ${ }^{1 *}$ \\ ${ }^{1}$ Department of Biological Sciences and Bioengineering, Indian Institute of Technology, Kanpur 208016 , \\ India \\ ${ }^{2}$ Present Address: Harvard Medical School and Brigham and Women's Hospital, Cambridge, USA \\ ${ }^{3}$ Institut de Génétique et de Biologie Moléculaire et Cellulare (IGBMC), Illkirch, France \\ *Corresponding author (Email, sganesh@iitk.ac.in) \\ published online 16 August 2018
}

Correction to: J. Biosci. 40(5), December 2015, 863-871 https://doi.org/10.1007/s12038-015-9570-0

The image of anti-Myc blot of figure 2C (third panel; Malin-Myc [C26S]) was inadvertently used once again for the $\gamma$-tubulin loading control of figure 2B. The revised figure 2B with the correct image of the $\gamma$-tubulin loading control is given below. The interpretation and conclusion provided in the article do not change because of the correction.

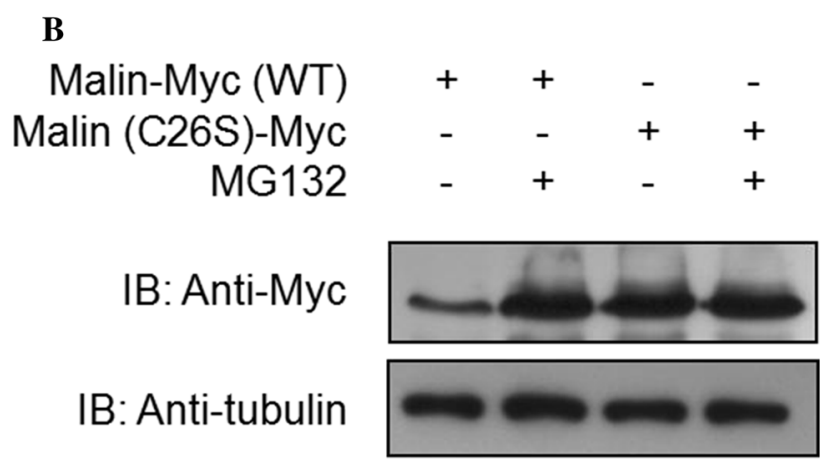

Figure 2. Malin promotes its own degradation: (A) The wild-type (WT) or the catalytically inactive (C26S) mutant malin was overexpressed in COS-7 cells and processed for pull-down assay using anti-ubiquitin antibody. The pull-down products (PD) and whole cell lysates (WCL) were resolved and immunoblotted (IB) with indicated antibodies. (B) Myc/His-tagged malin or Myc/His-tagged C26S malin was transiently expressed in COS-7 cells either in the presence or absence of MG132 as indicated. Equal amount of whole cell lysate for each combination was resolved in SDS-PAGE and immunoblotted with anti-Myc antibody or anti- $\gamma$-tubulin antibody (as loading control) to show the difference in the cellular levels of malin. (C) Transfections were done in COS-7 cells in 24-well plate for Myc-tagged wild-type malin or the Myc-tagged C26S mutant malin (300 ng/well) with increasing proportion of an expression construct (0, 100, 200, or $400 \mathrm{ng} /$ well in lanes 1 to 4, respectively) for the HA-tagged wild-type ubiquitin (WT) or its mutant (K48R) as indicated. Equal amount of whole cell lysate from each well was resolved in SDS-PAGE and immunoblotted with anti-Myc antibody or anti- $\gamma$-tubulin antibody (as loading control) to show the difference in the cellular levels of the proteins. 